\title{
Improved DAE formulation for inverse dynamics simulation of cranes
}

\author{
Wojciech Blajer • Krzysztof Kołodziejczyk
}

Received: 18 November 2009 / Accepted: 9 September 2010 / Published online: 1 October 2010

(C) The Author(s) 2010. This article is published with open access at Springerlink.com

\begin{abstract}
Cranes are underactuated systems with less control inputs than degrees of freedom. Dynamics and control of such systems is a challenging task, and the existence of solution to the inverse dynamics simulation problem in which an $r$-degree-of-freedom system with $m$ actuators, $m<r$, is subject to $m$ specified motion task (servo-constraints) is conditioned upon the system is differentially flat (all the system states and control inputs can be algebraically expressed in terms of the outputs and their time derivatives up to a certain order). The outputs are often designed as specified in time load coordinates to model a rest-to-rest maneuver along a trajectory in the working space, from the initial load position to its desired destination. The flatness-based methodology results then in the required control inputs determined in terms of the fourth time derivatives of the imposed outputs, and the derivations are featured by substantial complexity. The DAE formulation motivated in this contribution offers a more convenient approach to the prediction of dynamics and control of cranes executing prescribed load motions, and only the second time derivatives of the specified outputs are involved. While most of the inverse simulation formulations, both flatness-based and DAE ones, are performed using independent state variables, the use of dependent coordinates and velocities may lead to substantial modeling simplifications and gains in computational efficiency. An improved DAE formulation of this type is presented in this paper.
\end{abstract}

Keywords Inverse dynamics · Underactuated systems · Crane dynamics and control

\section{Introduction}

Cranes are underactuated systems with less control inputs than degrees of freedom; see, e.g., [1-6] for the backgrounds and other relevant technical examples. For underactuated systems, the inverse dynamics simulation problem in which an $r$-degree-of-freedom system with $m$

W. Blajer $(\bowtie) \cdot$ K. Kołodziejczyk

Faculty of Mechanical Engineering, Technical University of Radom, ul. Krasickiego 54, 26-600 Radom, Poland

e-mail: w.blajer@pr.radom.pl 
actuators, $m<r$, is subject to execute $m$ specified motion task, is a challenging task. While this problem is rather well understood for fully actuated systems whose motion is explicitly specified, $m=r$, with numerous applications to robot and process control, the existence of solution to the inverse simulation problem of underactuated systems is conditioned upon the system is differentially flat, which is explained in more detail in, e.g., [4] and [6]. All the system states and control inputs can then be algebraically expressed in terms of the outputs and their time derivatives up to a certain order.

In the modeling of a crane's conventional mode of operation, the desired outputs are commonly assumed as specified in time load coordinates that describe a rest-to-rest maneuver along a trajectory in the working space, from the initial load position to its desired destination [7]. The flatness-based methodology results in the required control inputs determined in terms of the fourth time derivatives of the imposed outputs, and the related derivations are featured by substantial complexity. The DAE formulation proposed in [5], and motivated in this contribution as well, offers an alternative and more convenient approach to the prediction of dynamics and control of cranes executing prescribed payload motions, where only the second time derivatives of the specified outputs are involved, and the solution consists in numerical estimation of crane's states and control inputs in the specified motion.

The mathematical models of cranes, used in both the flatness-based and DAE formulations of the inverse simulation studies, are typically built using minimal sets of independent coordinates [5, 7-12], leading to the minimal-dimension constraint reaction-free dynamic equations. The equations are of relative high complexity, however, assisted with further complexity of the so-called servo-constraints [12-14] or control constraints [5, 15] on the system, resulted from the specified motion requirements expressed in the independent variables. The final governing equations for the inverse dynamics analysis are then of rather elaborate structure. By contrast, the use of dependent (nonminimal) coordinates may lead to some simplifications in formulation of the problem, which was exploited in, e.g., [16] and [17] for the flatness based solution of control of kinematically undetermined cable suspension manipulators. In this paper, we adopt this idea to improve our previous DAE formulation [11] for the dynamics and control of cranes executing prescribed motions of payloads. In the dependent variable formulation, the robot (crane without the load) dynamics and the load dynamics are initially described separately, and are then coupled through a simple passive constraint due to the inextensible hoisting cable. Since the load motion is explicitly specified, the followed formulation of the inverse dynamics problem simplifies compared to the independent variable formulations [5, 10-12] in many modeling and computational issues. The advantages are emphasized in the sequel with reference to a three-dimensional rotary crane model executing a specified motion of the load.

\section{Modeling preliminaries}

Consider the three-dimensional rotary crane model seen in Fig. 1a, which constitutes a fivedegree-of-freedom $(r=5)$ system consisting of the girder, referenced further to as the rotating bridge (rotation $\varphi$, mass moment of inertia $J_{\mathrm{b}}$ with respect to the tower axis), the trolley (position $s$ on the bridge, mass $m_{\mathrm{t}}$ ), the winch (radius $r_{\mathrm{w}}$, moment of inertia $J_{\mathrm{w}}$ ), and the payload lumped with the hook and modeled as a point of mass $m_{1}$. It is assumed that the hoisting line is a longitudinally stiff and massless cable, and the winch radius $r_{\mathrm{w}}$ is negligible compared to the cable length $l$. The three $(m=3)$ control inputs are the torque $M_{\mathrm{b}}$ regulating the bridge rotation angle $\varphi$, the force $F$ actuating the trolley position $s$ on the bridge, and the winch torque $M_{\mathrm{w}}$ changing the rope length $l, \mathbf{u}=\left[\begin{array}{lll}M_{\mathrm{b}} & F & M_{\mathrm{w}}\end{array}\right]^{T}$. As $m<r$, 
a)

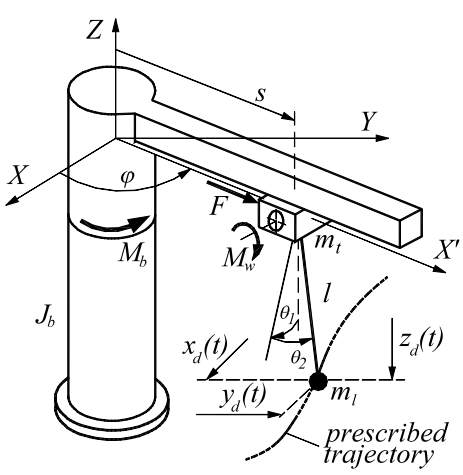

b)

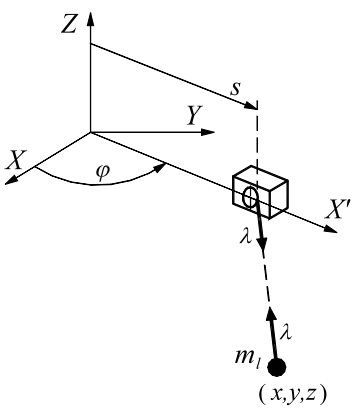

Fig. 1 The rotary crane model

the system at hand is underactuated, and the desired $m$ outputs of the system are specified in time load coordinates, $\mathbf{r}_{\mathrm{d}}(t)=\left[x_{\mathrm{d}}(t) y_{\mathrm{d}}(t) z_{\mathrm{d}}(t)\right]^{T}$.

The rotary crane position can be described using $r$ independent coordinates

$$
\mathbf{q}=\left[\varphi s l \theta_{1} \theta_{2}\right]^{T}
$$

where $\theta_{1}$ and $\theta_{2}$ are the swing angles defined in Fig. 1a. The other possibility is to use $n=r+1=2 m$ dependent coordinates

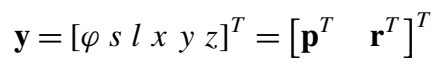

composed of $m$ robot (i.e., crane without the load) coordinates $\mathbf{p}=\left[\begin{array}{lll}\varphi & s & l\end{array}\right]^{T}$ and $m$ load coordinates $\mathbf{r}=\left[\begin{array}{lll}x & y & z\end{array}\right]^{T}$, related through the passive constraint (Fig. 1b)

$$
\Phi(\mathbf{y}) \equiv L-l=\sqrt{(x-s \cos \varphi)^{2}+(y-s \sin \varphi)^{2}+z^{2}}-l=0
$$

where $L(\varphi, s, x, y, z)$ defined above will be of some use in the sequel.

One important advantage of using the dependent coordinates $\mathbf{y}=\left[\mathbf{p}^{T} \mathbf{r}^{T}\right]^{T}$ is that the dynamic equations for the separated robot $\left(\mathbf{p}=\left[\begin{array}{ll}\varphi s & l\end{array}\right]^{T}\right)$ and load $\left(\mathbf{r}=\left[\begin{array}{ll}x & y \\ z\end{array}\right]^{T}\right)$ can easily be introduced in the following simple forms:

$$
\begin{aligned}
& \mathbf{M}_{\mathbf{p}}(\mathbf{p}) \ddot{\mathbf{p}}+\mathbf{d}_{\mathbf{p}}(\mathbf{p}, \dot{\mathbf{p}})=\mathbf{f}_{\mathbf{p}}(\dot{\mathbf{p}})-\mathbf{B}_{\mathbf{p}}^{T} \mathbf{u} \\
& \mathbf{M}_{\mathbf{r}} \ddot{\mathbf{r}}=\mathbf{f}_{\mathbf{r}}
\end{aligned}
$$

which, given explicitly, are respectively:

$$
\begin{aligned}
& {\left[\begin{array}{ccc}
J_{\mathrm{b}}+m_{\mathrm{t}} s^{2} & 0 & 0 \\
0 & m_{\mathrm{t}} & 0 \\
0 & 0 & J_{\mathrm{w}} / r_{\mathrm{w}}^{2}
\end{array}\right]\left[\begin{array}{c}
\ddot{\varphi} \\
\ddot{s} \\
\ddot{l}
\end{array}\right]+\left[\begin{array}{c}
2 s \dot{s} \dot{\varphi} \\
0 \\
0
\end{array}\right]} \\
& =\left[\begin{array}{c}
-D_{\varphi} \dot{\varphi} \\
-D_{\mathrm{s}} \dot{s} \\
-D_{\mathrm{l}} \dot{l}
\end{array}\right]-\left[\begin{array}{ccc}
-1 & 0 & 0 \\
0 & -1 & 0 \\
0 & 0 & -1 / r_{\mathrm{w}}
\end{array}\right]\left[\begin{array}{c}
M_{\mathrm{b}} \\
F \\
M_{\mathrm{w}}
\end{array}\right] \\
& {\left[\begin{array}{ccc}
m_{1} & 0 & 0 \\
0 & m_{1} & 0 \\
0 & 0 & m_{1}
\end{array}\right]\left[\begin{array}{c}
\ddot{x} \\
\ddot{y} \\
\ddot{z}
\end{array}\right]=\left[\begin{array}{c}
0 \\
0 \\
-m_{1} g
\end{array}\right]}
\end{aligned}
$$


where $g$ is the gravitational acceleration, and $D_{\varphi}, D_{\alpha}$, and $D_{\mathrm{s}}$ are the viscous damping coefficients related to the respective motions. Equations (4) and (5) can then be aggregated to formulate the crane dynamic equations

$$
\mathbf{M}(\mathbf{y}) \ddot{\mathbf{y}}+\mathbf{d}(\mathbf{y}, \dot{\mathbf{y}})=\mathbf{f}(\dot{\mathbf{y}})-\mathbf{B}^{T} \mathbf{u}-\mathbf{C}^{T}(\mathbf{y}) \lambda
$$

where $\mathbf{M}=\operatorname{diag}\left(\mathbf{M}_{\mathbf{p}}, \mathbf{M}_{\mathbf{r}}\right), \mathbf{d}=\left[\begin{array}{ll}\mathbf{d}_{\mathbf{p}}^{T} & \mathbf{0}^{T}\end{array}\right]^{T}, \mathbf{f}=\left[\begin{array}{l}\mathbf{f}_{\mathbf{p}}^{T} \mathbf{f}_{\mathbf{r}}^{T}\end{array}\right]^{T}, \mathbf{B}^{T}=\left[\begin{array}{l}\mathbf{B}_{\mathbf{p}}^{T} \vdots \mathbf{0}\end{array}\right]^{T}$, and $\lambda$ is the Lagrange multiplier related to the passive constraint of (3), which is the cable tension force seen in Fig. 1b, and $\mathbf{C}=\partial \Phi / \partial \mathbf{y}$ is the associated $1 \times n$ constraint matrix (Jacobian). By contrast, the crane dynamic equations in independent coordinates $\mathbf{q}$, whose symbolic form is $\mathbf{M}_{\mathbf{q}}(\mathbf{q}) \ddot{\mathbf{q}}+\mathbf{d}_{\mathbf{q}}(\mathbf{q}, \dot{\mathbf{q}})=\mathbf{f}_{\mathbf{q}}(\mathbf{q}, \dot{\mathbf{q}})-\mathbf{B}_{\mathbf{q}}^{T} \mathbf{u}$, are of much more complex explicit form and are much more laborious to derive (see $[10,11]$ for the details).

The only nuisance concerning the dependent coordinate formulation is derivation of the passive constraint conditions at the velocity and acceleration levels:

$$
\begin{aligned}
\dot{\Phi} & \equiv \mathbf{C}(\mathbf{y}) \dot{\mathbf{y}}=0, \\
\ddot{\Phi} & \equiv \mathbf{C}(\mathbf{y}) \ddot{\mathbf{y}}-\xi(\mathbf{y}, \dot{\mathbf{y}})=0
\end{aligned}
$$

While the $1 \times n$ passive constraint matrix $\mathbf{C}=\partial \Phi / \partial \mathbf{y}$ is

$$
\mathbf{C}=\left[\begin{array}{llllll}
\frac{(x \sin \varphi-y \cos \varphi) s}{L} & \frac{s-x \cos \varphi-y \sin \varphi}{L} & -1 & \frac{x-s \cos \varphi}{L} & \frac{y-s \sin \varphi}{L} & \frac{z}{L}
\end{array}\right]
$$

where, as defined in (3), $L=\sqrt{(x-s \cos \varphi)^{2}+(y-s \sin \varphi)^{2}+z^{2}}$, the derivation of the constraint-induced acceleration $\xi=-\dot{\mathbf{C}} \dot{\mathbf{y}}$ in an analytical form is somewhat more demanding, mainly due to $L(\varphi, s, x, y, z)$ appears in the denominators of $\mathbf{C}$ elements. Some simplifications can, however, be achieved by introducing $\mathbf{C}^{*}=\mathbf{C} L$ (with no denominators in $\mathbf{C}^{*}$ ). Then, since $\dot{\mathbf{C}}^{*}=\dot{\mathbf{C}} L+\mathbf{C} \dot{L}$, the constraint-induced acceleration $\xi$ is

$$
\xi=-\dot{\mathbf{C}} \dot{\mathbf{y}}=\left(\mathbf{C} \dot{L}-\dot{\mathbf{C}}^{*}\right) \dot{\mathbf{y}} / L
$$

Both $\dot{L}$ and $\dot{\mathbf{C}}^{*}$ are relatively easy to derive compared to the direct derivation of $\dot{\mathbf{C}}$. Alternatively, one can also formulate the passive constraint equation in a more tractable way as $\Phi^{\prime}(\mathbf{y})=(x-s \cos \varphi)^{2}+(y-s \sin \varphi)^{2}+z^{2}-l^{2}=0$, which leads to substantial simplifications in $\mathbf{C}^{\prime}=\partial \Phi^{\prime} / \partial \mathbf{y}$ and $\xi^{\prime}=-\dot{\mathbf{C}}^{\prime} \dot{\mathbf{y}}$. The generalized constraint reaction vector $\mathbf{f}_{\mathrm{c}}=-\mathbf{C}^{T}(\mathbf{y}) \lambda$ in (10) should then be replaced with $\mathbf{f}_{\mathrm{c}}=-\mathbf{C}^{\prime T}(\mathbf{y}) \lambda^{\prime}$. However, while $\lambda$ denotes the tensile force in the cable (its dimension is $\mathrm{kgm} / \mathrm{s}^{2}$ ), the Lagrange multiplier $\lambda^{\prime}$ has no denotation of a physical force (its dimension is $\mathrm{kg} / \mathrm{s}^{2}$ ), and obtainment of the cable tensile force from $\lambda^{\prime}$ is not straightforward. This is why the cable length condition is recommended to be formulated in the "length" form (3), and not in the "square length" form.

The crane performance goal is to execute a prescribed motion of the load. The desired $m=3$ outputs of the crane are s pecified in time load coordinates,

$$
\mathbf{r}_{\mathrm{d}}(t)=\left[\begin{array}{lll}
x_{\mathrm{d}}(t) & y_{\mathrm{d}}(t) & z_{\mathrm{d}}(t)
\end{array}\right]^{T}
$$

which can conveniently be defined by setting a straight line or curvilinear trajectory $\widehat{\mathbf{r}}_{\mathrm{d}}(s)$ in the crane working space (from the initial load position to its desired destination), and then imposing an appropriately smooth reference function $s(t)$ that describes the load motion along the trajectory, $\mathbf{r}_{\mathrm{d}}(t)=\widehat{\mathbf{r}}_{\mathrm{d}}[s(t)]$. Such procedures for the load motion planning, patterned on the propositions posed in [18], were described in detail in [19]. 
The time-specified system outputs lead to servo-constraints [12-14] (or control constraints $[5,15])$ on the system. Using the dependent coordinates $\mathbf{y}=\left[\mathbf{p}^{T} \mathbf{r}^{T}\right]^{T}$, the $m$ servoconstraint equations simplify to the following trivial form

$$
\boldsymbol{\Psi}(\mathbf{y}, t) \equiv \mathbf{r}-\mathbf{r}_{\mathrm{d}}(t)=0
$$

and the resultant $m \times n$ servo-constraint matrix $\mathbf{S}=\partial \boldsymbol{\Psi} / \partial \mathbf{y}$ is

$$
\mathbf{S}=[\mathbf{0} \vdots \mathbf{I}]=\left[\begin{array}{llllll}
0 & 0 & 0 & 1 & 0 & 0 \\
0 & 0 & 0 & 0 & 1 & 0 \\
0 & 0 & 0 & 0 & 0 & 1
\end{array}\right]
$$

where $\mathbf{0}$ and $\mathbf{I}$ are the zero and identity matrices of dimension $m \times m$. As shown in $[5,10-12]$, the use of independent coordinates $\mathbf{q}$ results in the much more complex servoconstraint equations, which must then be twice differentiated with respect to time (further substantial complexity) when involved in the governing equations for the inverse dynamics analysis. The present formulation is free from those demanding derivation tasks. The other profit of using $\mathbf{y}=\left[\mathbf{p}^{T} \mathbf{r}^{T}\right]^{T}$ is that the load coordinates $\mathbf{r}$, being explicitly specified by $\mathbf{r}=\mathbf{r}_{\mathrm{d}}(t)$, can be excluded from the unknowns in the inverse simulation study. Assumed $\mathbf{r}_{\mathrm{d}}(t)$ are appropriately smooth functions, explicitly specified are also the load velocities and accelerations, $\dot{\mathbf{r}}=\dot{\mathbf{r}}_{\mathrm{d}}(t)$ and $\ddot{\mathbf{r}}=\ddot{\mathbf{r}}_{\mathrm{d}}(t)$.

\section{Governing equations}

The initial governing equations for the inverse dynamics analysis of the crane executing the load prescribed motion can be formulated as the following set of $2 n+m+1=5 m+1$ differential-algebraic equations (DAEs)

$$
\begin{aligned}
\dot{\mathbf{y}} & =\mathbf{w} \\
\mathbf{M}(\mathbf{y}) \dot{\mathbf{w}} & =\mathbf{f}(\mathbf{w})-\mathbf{d}(\mathbf{y}, \mathbf{w})-\mathbf{B}^{T} \mathbf{u}-\mathbf{C}^{T}(\mathbf{y}) \lambda \\
\mathbf{0} & =\boldsymbol{\Psi}(\mathbf{y}, t) \\
0 & =\Phi(\mathbf{y})
\end{aligned}
$$

in $2 n$ states $\mathbf{y}$ and $\mathbf{w}, m$ control inputs $\mathbf{u}$, and the Lagrange multiplier $\lambda$.

An important characteristic of a DAE system is its index, which is a measure of singularity of DAEs and indicates 'how far' the DAE system is from an equivalent set of ODEs (ordinary differential-equations) [20]. Roughly speaking, the index of a DAE system is the number of times one needs to differentiate the algebraic equations to get an equivalent system of differential equations [21]. The index of the initial governing DAEs gathered in (14) is equal to five [5], which yields difficulties in their direct numerical treatment (most commercial DAE solvers are designed for index-one DAEs [22, 23]). Therefore, it is desirable to transform the above initial index-five DAEs into an equivalent, numerically more tractable form of index-three DAEs. The present index reduction procedure described below is qualitatively similar to that proposed in [5] for the independent variable formulation.

Before going further, it may be worth to look on the problem at hand from the geometrical point of view; see also [24] for more geometrical insight. For the present formulation of crane dynamics in the dependent coordinates $\mathbf{y}$, the linear $n$-space $\mathcal{Y}$ related to $\dot{\mathbf{y}}, n=2 m$, 
Fig. 2 The subspace $\mathcal{Y}$ and the related subspaces

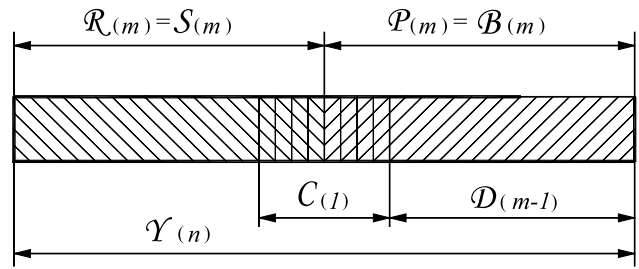

is split into two complementary and disjoint $m$-subspaces: the robot subspace $\mathcal{P}$ (related to $\dot{\mathbf{p}}$ ) and the load subspace $\mathcal{R}$ (related to $\dot{\mathbf{r}}$ ), $\mathcal{P} \cup \mathcal{R}=\mathcal{Y}$ and $\mathcal{P} \cap \mathcal{R}=0$, which is illustrated symbolically in Fig. 2. The $\mathcal{P}$ and $\mathcal{R}$ subspaces are equivalent, respectively, to the controlled subspace $\mathcal{B}$ spanned by the vectors represented as rows in $\mathbf{B}$ and to the specified subspace $\mathcal{S}$ spanned by the vectors represented as rows in $\mathbf{S}, \mathcal{P}=\mathcal{B}$ and $\mathcal{R}=\mathcal{S}$. There exists also a onedimensional constrained subspace $\mathcal{C}$, spanned by the passive constraint gradient represented in $\mathbf{C}$. The constrained subspace $\mathcal{C}$ has a nonzero inner product with both $\mathcal{P}=\mathcal{B}$ and $\mathcal{R}=\mathcal{S}$ subspaces, i.e. $\mathcal{C} \cap \mathcal{P} \neq 0(\mathcal{C} \cap \mathcal{B} \neq 0)$ and $\mathcal{C} \cap \mathcal{R} \neq 0(\mathcal{C} \cap \mathcal{S} \neq 0)$, which reflects the fact that the passive constraint defined in (3) couples the robot and load motions, and the physical representation of the coupling is the constraint reaction $\lambda$ (the cable tension force). The dimension of subspace $\mathcal{S} \cup \mathcal{C}$ is then $m+1$, and its complement in $\mathcal{N}$ is the $m-1$-dimensional tangential subspace $\mathcal{D}$ in which the crane motion is neither specified nor constrained, i.e., $(\mathcal{S} \cup \mathcal{C}) \cup \mathcal{C}=\mathcal{Y}$ and $(\mathcal{S} \cup \mathcal{C}) \cap \mathcal{D}=0$. The latter condition shows that the $\mathcal{D}$ subspace can be defined by $m-1$ vectors contained as columns in an $n \times(m-1)$ matrix $\mathbf{D}$ such that

$$
\left[\begin{array}{l}
\mathbf{S} \\
\mathbf{C}
\end{array}\right] \mathbf{D}=\mathbf{0} \quad \Leftrightarrow \quad \begin{aligned}
& \mathbf{S D}=\mathbf{0} \\
& \mathbf{C D}=\mathbf{0}
\end{aligned} \Rightarrow \begin{aligned}
& \mathbf{D}^{T} \mathbf{S}^{T}=\mathbf{0} \\
& \mathbf{D}^{T} \mathbf{C}^{T}=\mathbf{0}
\end{aligned}
$$

Matrix $\mathbf{D}$ is thus an orthogonal complement matrix to the $(m+1) \times n$-dimensional matrix $\left[\mathbf{S}^{T} \vdots \mathbf{C}^{T}\right]^{T}$. For the rotary crane model, owing to the trivial structure of $\mathbf{S}$ defined in (13), the $6 \times 2$ matrix $\mathbf{D}$ can be found by inspection, i.e.,

$$
\mathbf{D}^{T}=\left[\begin{array}{llllll}
1 & 0 & C_{1} & 0 & 0 & 0 \\
0 & 1 & C_{2} & 0 & 0 & 0
\end{array}\right]
$$

where $C_{1}$ and $C_{2}$ are the first and the second entries of $\mathbf{C}$ defined in (9). The simplicity in obtaining $\mathbf{D}$ is another triumph of the dependent variable formulation, which is not the case of the independent coordinate formulation (see $[10,11]$ for the details).

The mentioned index reduction procedure of the governing DAEs reported in (14) consists first in projecting the dynamic equations $(14)_{2}$ into, respectively, the tangential subspace $\mathcal{D}$, the specified subspace $\mathcal{S}$, and the constrained subspace $\mathcal{C}$. The projection is then associated with the substitution of $\mathbf{r}_{\mathrm{d}}(t), \dot{\mathbf{r}}_{\mathrm{d}}(t)$, and $\ddot{\mathbf{r}}_{\mathrm{d}}(t)$ instead of $\mathbf{r}, \dot{\mathbf{r}}$ and $\ddot{\mathbf{r}}$, respectively, which allows one to exclude the load states from the unknowns in the subsequent inverse simulation study. With these simplifications and some other rearrangements, the projection of the dynamic equations into $\mathcal{D}, \mathcal{S}$, and $\mathcal{C}$ is represented in matrix notation by (see [24] for the background)

$$
\left[\begin{array}{c}
\mathbf{D}^{T}(\mathbf{p}, t) \\
\mathbf{S M}^{-1} \\
\mathbf{C}(\mathbf{p}, t) \mathbf{M}^{-1}
\end{array}\right]\left(\left[\begin{array}{c}
\mathbf{M}_{\mathbf{p}} \ddot{\mathbf{p}} \\
\mathbf{M}_{\mathbf{r}} \ddot{\mathbf{r}}_{\mathrm{d}}(t)
\end{array}\right]+\left[\begin{array}{c}
\mathbf{d}_{\mathbf{p}}(\mathbf{p}, \dot{\mathbf{p}}) \\
\mathbf{0}
\end{array}\right]-\left[\begin{array}{c}
\mathbf{f}_{\mathbf{p}}(\dot{\mathbf{p}}) \\
\mathbf{f}_{\mathbf{r}}
\end{array}\right]+\left[\begin{array}{c}
\mathbf{B}_{\mathbf{p}}^{T} \\
\mathbf{0}
\end{array}\right] \mathbf{u}+\mathbf{C}^{T}(\mathbf{p}, t) \lambda\right)=\mathbf{0}
$$

where $\mathbf{D}(\mathbf{y})=\mathbf{D}\left(\mathbf{p}, \mathbf{r}_{\mathrm{d}}(t)\right)=\mathbf{D}(\mathbf{p}, t)$ and $\mathbf{C}(\mathbf{y})=\mathbf{C}\left(\mathbf{p}, \mathbf{r}_{\mathrm{d}}(t)\right)=\mathbf{C}(\mathbf{p}, t)$. 
Taking into account that, according to (15), $\mathbf{D}^{T} \mathbf{C}^{T}=\mathbf{0}$, and then, according to (16), $\mathbf{D}^{T}=\left[\mathbf{D}_{\mathbf{p}}^{T} \vdots \mathbf{0}\right]$, the projection into $\mathcal{D}$ leads to the following $m-1=2$ differential equations

$$
\mathbf{D}_{\mathbf{p}}^{T} \mathbf{M}_{\mathbf{p}} \ddot{\mathbf{p}}+\mathbf{D}_{\mathbf{p}}^{T}\left(\mathbf{d}_{\mathbf{p}}-\mathbf{f}_{\mathbf{p}}\right)+\mathbf{D}_{\mathbf{p}}^{T} \mathbf{B}_{\mathbf{p}} \mathbf{u}=\mathbf{0} \quad \Leftrightarrow \quad \mathbf{H}(\mathbf{p}, t) \ddot{\mathbf{p}}+\mathbf{h}(\mathbf{p}, \dot{\mathbf{p}}, \mathbf{u}, t)=\mathbf{0}
$$

The projection into $\mathcal{S}$, considering that $\mathbf{S}=[\mathbf{0} \vdots \mathbf{I}]$ and $\mathbf{M}^{-1}=\operatorname{diag}\left(\mathbf{M}_{\mathbf{p}}^{-1}, \mathbf{M}_{\mathbf{r}}^{-1}\right)$, results then in the following $m$ algebraic equations

$$
\ddot{\mathbf{r}}_{\mathrm{d}}-\mathbf{M}_{\mathbf{r}}^{-1} \mathbf{f}_{\mathbf{r}}+\mathbf{M}_{\mathbf{r}}^{-1} \mathbf{C}_{\mathbf{r}}^{T} \lambda=\mathbf{0} \quad \Leftrightarrow \quad \mathbf{s}(\mathbf{p}, \lambda, t)=\mathbf{0}
$$

where $\mathbf{C}_{\mathbf{r}}$ denotes the last $m$ entries of $\mathbf{C}$. Finally, the projection into $\mathcal{C}$, after replacing $\mathbf{C} \ddot{\mathbf{y}}$ with $\xi(\mathbf{y}, \dot{\mathbf{y}})=\xi\left(\mathbf{p}, \mathbf{r}_{\mathrm{d}}(t), \dot{\mathbf{p}}, \dot{\mathbf{r}}_{\mathrm{d}}(t)\right)=\xi(\mathbf{p}, \dot{\mathbf{p}}, t)$, results in one equation

$$
\xi+\mathbf{C M}^{-1}(\mathbf{d}-\mathbf{f})+\mathbf{C M}^{-1} \mathbf{B}^{T} \mathbf{u}+\mathbf{C M}^{-1} \mathbf{C}^{T} \lambda=\mathbf{0} \quad \Leftrightarrow \quad c(\mathbf{p}, \dot{\mathbf{p}}, \mathbf{u}, \lambda, t)=0
$$

Using (18)-(20), the final reduced-dimension governing equations for the inverse dynamics analysis are the following $m+(m-1)+m+1+1=3 m+1=10$ index-three DAEs in $\mathbf{p}, \dot{\mathbf{p}}=\mathbf{v}, \mathbf{u}$, and $\lambda$ :

$$
\begin{aligned}
\dot{\mathbf{p}}-\mathbf{v} & =\mathbf{0} \\
\mathbf{H}(\mathbf{p}, t) \dot{\mathbf{v}}+\mathbf{h}(\mathbf{p}, \mathbf{v}, \mathbf{u}, t) & =\mathbf{0} \\
\mathbf{s}(\mathbf{p}, \lambda, t) & =\mathbf{0} \\
c(\mathbf{p}, \mathbf{v}, \mathbf{u}, \lambda, t) & =0 \\
\Phi(\mathbf{p}, t) & =0
\end{aligned}
$$

The solution to the above DAEs are the variations in time of the robot state variables that ensure execution of the load prescribed motion, $\mathbf{p}_{\mathrm{d}}(t)$ and $\mathbf{v}_{\mathrm{d}}(t)$, the control variations $\mathbf{u}_{\mathrm{d}}(t)$ required to enforce the robot motion, and the resultant cable tension force variations $\lambda_{\mathrm{d}}(t)$. The solution covers thus both the dynamic analysis of motion of the underactuated system in the partly specified motion as well as the determination of the required control inputs.

It may be worth to note that the reduction in dimension of the above DAEs is mainly due to the fact that the servo-constraint conditions as well as the load state variables are excluded from the analysis. This cannot be achieved when using the independent coordinates $\mathbf{q}$. The servo-constraint equations expressed in these coordinates must be included in the respective governing DAEs, whose number, for the sample rotary crane $[10,11]$, is $2 r+m=$ $5 m-1=14$, compared to the number $3 m+1=10$ of the present governing DAEs. The governing DAEs formulated in $\mathbf{q}$ are also inherently more complex compared to the present ones. An additional advantage of the present formulation is that the cable tension force $\lambda$ is involved, which makes it possible to monitor its value during the simulated maneuvers. This may be of importance since the passive constraint of (3) is unilateral, and $\lambda$ should never become negative. In the independent coordinate formulation, the passive constraint (and as such $\lambda$ ) are eliminated from the analysis, and are treated as bilateral constraints in the elimination procedure. 


\section{Solution codes}

The explicit forms of $m=3$ algebraic equations $(21)_{3}, \mathbf{s}(\mathbf{p}, \lambda, t)=\mathbf{0}$, followed from the projection of the dynamic equations in the specified subspace are:

$$
\ddot{x}_{\mathrm{d}}+\frac{x_{\mathrm{d}}-s \cos \varphi}{m_{\mathrm{l}} L} \lambda=0 ; \quad \ddot{y}_{\mathrm{d}}+\frac{y_{\mathrm{d}}-s \sin \varphi}{m_{\mathrm{l}} L} \lambda=0 ; \quad \ddot{z}_{\mathrm{d}}+g+\frac{z_{\mathrm{d}}}{m_{\mathrm{l}} L} \lambda=0
$$

where $L^{2}=\left(x_{\mathrm{d}}-s \cos \varphi\right)^{2}+\left(y_{\mathrm{d}}-s \sin \varphi\right)^{2}+z_{\mathrm{d}}^{2}$. These are three nonlinear equations in $s, \varphi$, and $\lambda$, from which, after some manipulations, one obtains:

$$
\lambda_{\mathrm{d}}(t)=m_{\mathrm{l}} \sqrt{\ddot{x}_{\mathrm{d}}^{2}+\ddot{y}_{\mathrm{d}}^{2}+\left(\ddot{z}_{\mathrm{d}}+g\right)^{2}} ; \quad s_{\mathrm{d}}(t)=\sqrt{A_{x}^{2}+A_{y}^{2}} ; \quad \varphi_{\mathrm{d}}(t)=\arctan \left(A_{y} / A_{x}\right)
$$

where $A_{x}=x_{\mathrm{d}}-z_{\mathrm{d}} \ddot{x}_{\mathrm{d}} /\left(\ddot{z}_{\mathrm{d}}+g\right)$ and $A_{y}=y_{\mathrm{d}}-z_{\mathrm{d}} \ddot{y}_{\mathrm{d}} /\left(\ddot{z}_{\mathrm{d}}+g\right)$, and then, from the passive constraint condition of $(21)_{5}$ or (3), it comes that

$$
l_{\mathrm{d}}(t)=z_{\mathrm{d}} \frac{\sqrt{\ddot{x}_{\mathrm{d}}^{2}+\ddot{y}_{\mathrm{d}}^{2}+\left(\ddot{z}_{\mathrm{d}}+g\right)^{2}}}{\ddot{z}_{\mathrm{d}}+g}
$$

The above analytical solutions for $\lambda_{\mathrm{d}}(t)$ and $\mathbf{p}_{\mathrm{d}}(t)$ are a part of the flatness-based solution to the differentially flat problem of the inverse dynamics analysis for the crane executing the prescribed load motion, denoted that all the system state variables and control inputs can be algebraically expressed in terms of the desired outputs and their time derivatives up to a certain order (see, e.g., [4] and [6] for the theoretical background of differential flatness). For the case at hand, the general symbolic form of this flatness-based solution is [12]

$$
\begin{aligned}
& \lambda_{\mathrm{d}}(t)=\lambda\left(\mathbf{r}_{\mathrm{d}}, \dot{\mathbf{r}}_{\mathrm{d}}, \ddot{\mathbf{r}}_{\mathrm{d}}\right), \quad \mathbf{p}_{\mathrm{d}}(t)=\mathbf{p}\left(\mathbf{r}_{\mathrm{d}}, \dot{\mathbf{r}}_{\mathrm{d}}, \ddot{\mathbf{r}}_{\mathrm{d}}\right), \\
& \mathbf{v}_{\mathrm{d}}(t)=\mathbf{v}\left(\mathbf{r}_{\mathrm{d}}, \dot{\mathbf{r}}_{\mathrm{d}}, \ddot{\mathbf{r}}_{\mathrm{d}}, \mathbf{r}_{\mathrm{d}}^{(3)}\right), \\
& \mathbf{u}_{\mathrm{d}}(t)=\mathbf{u}\left(\mathbf{r}_{\mathrm{d}}, \dot{\mathbf{r}}_{\mathrm{d}}, \ddot{\mathbf{r}}_{\mathrm{d}}, \mathbf{r}_{\mathrm{d}}^{(3)}, \mathbf{r}_{\mathrm{d}}^{(4)}\right) .
\end{aligned}
$$

As seen in (23) and (24), the solutions $\lambda_{\mathrm{d}}(t)$ and $\mathbf{p}_{\mathrm{d}}(t)$ depend on the specified outputs $\mathbf{r}_{\mathrm{d}}(t)$ and their time derivatives up to the second order (actually, they do not depend here on $\dot{\mathbf{r}}_{\mathrm{d}}$ ). Further differentiation with respect to time of the algebraic equations of governing DAEs reported in (21), combined with (extremely complex) algebraic manipulations, would then lead, firstly (after the first time-differentiation), to the algebraic expression $(25)_{2}$ for $\mathbf{v}_{\mathbf{d}}(t)$, and then (after the second time-differentiation), to the algebraic expression $(25)_{3}$ for the required control inputs $\mathbf{u}_{\mathrm{d}}(t)$. The latter solution will depend on the specified outputs and their time derivatives up to $\alpha=4$ order, and $\alpha$ is by one smaller than the index $(i=5)$ of the initial governing DAEs reported in (14). The flatness-based solutions were demonstrated in [16] for a 2D overhead crane model, and in [17] for a 3D cable suspension manipulator.

The flatness-based solutions of (25), especially those for $\mathbf{v}_{\mathrm{d}}(t)$ and $\mathbf{u}_{\mathrm{d}}(t)$, are featured by enormous complexity, and, as such, they may be considered as impractical in applications. Using the governing index-three DAEs in the form of (19), and solving them numerically is much more straightforward and applicable. The solution methodology proposed in [5] can directly be applied to the present DAEs. The numerical code is based on Euler backward differentiation scheme, in which the time derivatives $\dot{\mathbf{p}}$ and $\dot{\mathbf{v}}$ are approximated with their backward differences, respectively, $\left(\mathbf{p}_{k+1}-\mathbf{p}_{k}\right) / \Delta t$ and $\left(\mathbf{v}_{k+1}-\mathbf{v}_{k}\right) / \Delta t$, where $\Delta t$ is the integration time step. Given $\mathbf{p}_{k}$ and $\mathbf{v}_{k}$ at time $t_{k}\left(\mathbf{u}_{k}\right.$ and $\lambda_{k}$ are not involved), the values 
$\mathbf{p}_{k+1}, \mathbf{v}_{k+1}, \mathbf{u}_{k+1}$ and $\lambda_{k+1}$ at time $t_{k+1}=t_{k}+\Delta t$ can be determined as a solution to the following nonlinear algebraic equations:

$$
\begin{aligned}
\mathbf{p}_{k+1}-\mathbf{p}_{k}-\Delta t \mathbf{v}_{k+1} & =\mathbf{0} \\
\mathbf{H}\left(\mathbf{p}_{k+1}, t_{k+1}\right)\left(\mathbf{v}_{k+1}-\mathbf{v}_{k}\right)+\Delta t \mathbf{h}\left(\mathbf{p}_{k+1}, \mathbf{v}_{k+1}, \mathbf{u}_{k+1}, t_{k+1}\right) & =\mathbf{0} \\
\mathbf{s}\left(\mathbf{p}_{k+1}, \lambda_{k+1}, t_{k+1}\right) & =\mathbf{0} \\
c\left(\mathbf{p}_{k+1}, \mathbf{v}_{k+1}, \mathbf{u}_{k+1}, \lambda_{k+1}, t_{k+1}\right) & =0 \\
\Phi\left(\mathbf{p}_{k+1}, t_{k+1}\right) & =0
\end{aligned}
$$

and in this way the solution is advanced from $t_{k}$ to $t_{k+1}=t_{k}+\Delta t$. As motivated in $[5,12]$, the numerical solutions for $\mathbf{p}_{\mathrm{d}}(t)$ and $\lambda_{\mathrm{d}}(t)$ obtained from (26), which corresponds to the analytical solutions of (23) and (24), are exact with numerical accuracy. The rough backward difference scheme influences only the accuracy of determination of $\mathbf{v}_{\mathrm{d}}(t)$ and $\mathbf{u}_{\mathrm{d}}(t)$. The truncation errors do not accumulate in time, however, as the approximations for $\mathbf{v}_{k+1}$ and $\mathbf{u}_{k+1}$ at time $t_{k+1}$ are always based on the numerically exact values $\mathbf{p}_{k}, \mathbf{p}_{k+1}$, and $\lambda_{k+1}$. The above simple (and effective) scheme leads thus to stable solutions. More details concerned with the proposed solution procedure can be found in $[5,11]$.

\section{Case study}

The rotary crane data used in calculations were: $J_{\mathrm{b}}=30000 \mathrm{~kg} \mathrm{~m}^{2}, m_{\mathrm{t}}=50 \mathrm{~kg}$, $m_{1}=500 \mathrm{~kg}, J_{\mathrm{w}}=0.1 \mathrm{~kg} \mathrm{~m}^{2}, r_{\mathrm{w}}=0.1 \mathrm{~m}$, and the damping coefficients $D_{\varphi}, D_{\mathrm{s}}$, and $D_{\mathrm{l}}$ were all set to zero for simplicity. The specified trajectory of the load is illustrated in Fig. 3, with the initial load position at $(14 \mathrm{~m}, 0 \mathrm{~m},-15 \mathrm{~m})$ and the destination point in $(0 \mathrm{~m},-5 \mathrm{~m},-3 \mathrm{~m})$. In order to avoid the obstacle seen in the figure as well as not to transport the load too close to the tower, the load motion was initially modeled in cylindrical coordinates $\gamma=\left[\begin{array}{lll}r & \phi & z\end{array}\right]^{T}$, i.e.,

$$
\gamma_{\mathrm{d}}(t)=\gamma_{0}+\left(\gamma_{\mathrm{f}}-\gamma_{0}\right) s(t) ; \quad \dot{\gamma}_{\mathrm{d}}(t)=\left(\gamma_{\mathrm{f}}-\gamma_{0}\right) \dot{s}(t) ; \quad \ddot{\gamma}_{\mathrm{d}}(t)=\left(\gamma_{\mathrm{f}}-\gamma_{0}\right) \ddot{s}(t)
$$

where $\gamma_{0}=\left[\begin{array}{lll}r_{0} & \phi_{0} & z_{0}\end{array}\right]^{T}$ and $\gamma_{\mathrm{f}}=\left[\begin{array}{lll}r_{\mathrm{f}} & \phi_{\mathrm{f}} & z_{\mathrm{f}}\end{array}\right]^{T}$ are the initial and destination load positions in these coordinates, $r_{0}=15 \mathrm{~m}, r_{\mathrm{f}}=5 \mathrm{~m}, \phi_{0}=0 \mathrm{deg}, \phi_{\mathrm{f}}=270 \mathrm{deg}, z_{0}=-15 \mathrm{~m}$ and $z_{\mathrm{f}}=$ $-3 \mathrm{~m}$. The load motion specification introduced in $(11), \mathbf{r}_{\mathrm{d}}(t)=\left[x_{\mathrm{d}}(t) y_{\mathrm{d}}(t) z_{\mathrm{d}}(t)\right]^{T}$, and the first and the second derivatives with respect to time of $\mathbf{r}_{\mathrm{d}}(t)$, are then determined as:

$$
\begin{aligned}
& \mathbf{r}_{\mathrm{d}}(t)=\left[\begin{array}{c}
x_{\mathrm{d}}(t) \\
y_{\mathrm{d}}(t) \\
z_{\mathrm{d}}(t)
\end{array}\right]=\left[\begin{array}{c}
r_{\mathrm{d}} \cos \varphi_{\mathrm{d}} \\
r_{\mathrm{d}} \sin \varphi_{\mathrm{d}} \\
z_{\mathrm{d}}
\end{array}\right] \\
& \dot{\mathbf{r}}_{\mathrm{d}}(t)=\left[\begin{array}{c}
\dot{x}_{\mathrm{d}}(t) \\
\dot{y}_{\mathrm{d}}(t) \\
\dot{z}_{\mathrm{d}}(t)
\end{array}\right]=\left[\begin{array}{ccc}
\cos \varphi_{\mathrm{d}} & -r_{\mathrm{d}} \sin \varphi_{\mathrm{d}} & 0 \\
\sin \varphi_{\mathrm{d}} & r_{\mathrm{d}} \cos \varphi_{\mathrm{d}} & 0 \\
0 & 0 & 1
\end{array}\right]\left[\begin{array}{c}
\dot{r}_{\mathrm{d}} \\
\dot{\varphi}_{\mathrm{d}} \\
\dot{z}_{\mathrm{d}}
\end{array}\right] \\
& \ddot{\mathbf{r}}_{\mathrm{d}}(t)=\left[\begin{array}{c}
\ddot{x}_{\mathrm{d}}(t) \\
\ddot{y}_{\mathrm{d}}(t) \\
\ddot{z}_{\mathrm{d}}(t)
\end{array}\right]=\left[\begin{array}{ccc}
\cos \varphi_{\mathrm{d}} & -r_{\mathrm{d}} \sin \varphi_{\mathrm{d}} & 0 \\
\sin \varphi_{\mathrm{d}} & r_{\mathrm{d}} \cos \varphi_{\mathrm{d}} & 0 \\
0 & 0 & 1
\end{array}\right]\left[\begin{array}{c}
\ddot{r}_{\mathrm{d}} \\
\ddot{\varphi}_{\mathrm{d}} \\
\ddot{z}_{\mathrm{d}}
\end{array}\right]+\left[\begin{array}{c}
-2 \dot{r}_{\mathrm{d}} \dot{\varphi}_{\mathrm{d}} \sin \varphi_{\mathrm{d}}-r_{\mathrm{d}} \dot{\varphi}_{\mathrm{d}}^{2} \cos \varphi_{\mathrm{d}} \\
2 \dot{r}_{\mathrm{d}} \dot{\varphi}_{\mathrm{d}} \cos \varphi_{\mathrm{d}}+r_{\mathrm{d}} \dot{\varphi}_{\mathrm{d}}^{2} \sin \varphi_{\mathrm{d}} \\
0
\end{array}\right]
\end{aligned}
$$

Following the idea posed in [18], the reference function $s(t)$ was designed so that to divide the maneuver into the acceleration $(I)$, steady velocity $(I I)$, and deceleration $(I I I)$ phases. 
Fig. 3 The specified trajectory of the load
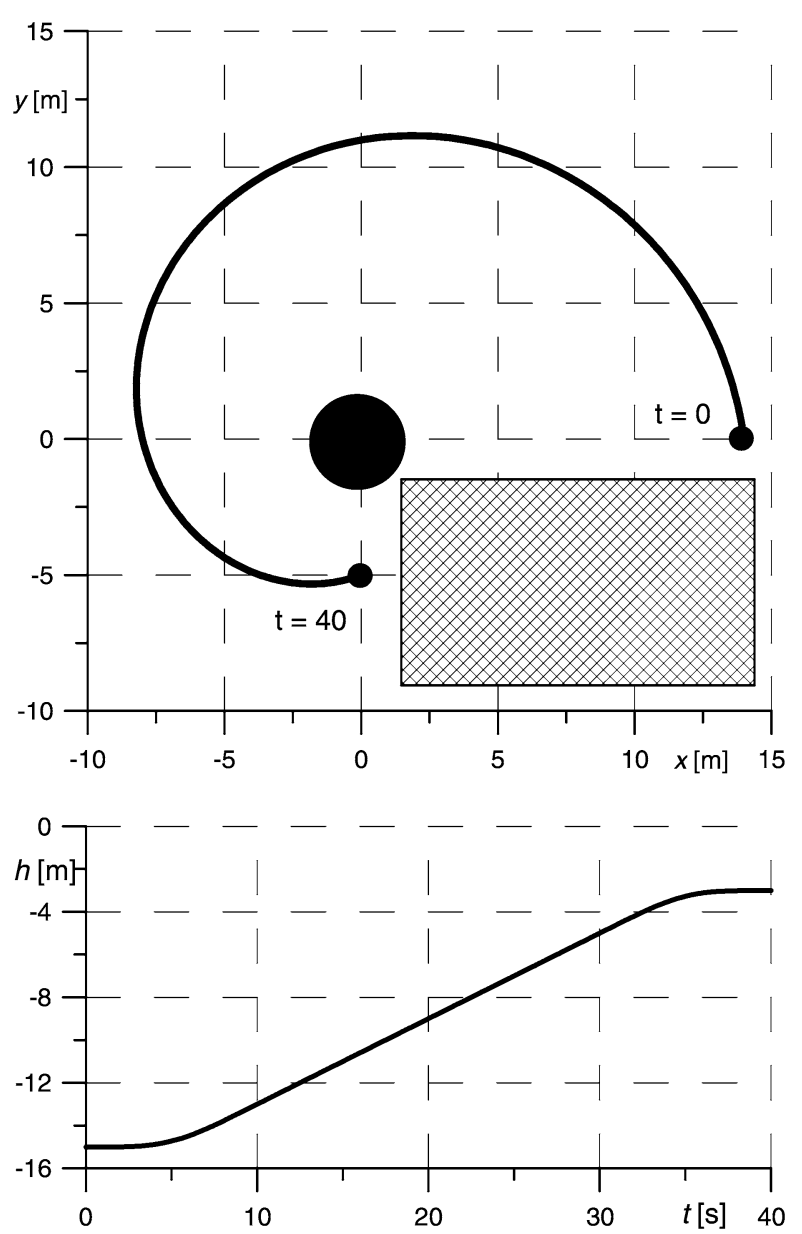

For $\tau=t_{\mathrm{f}}-t_{0}$ and the same acceleration and deceleration time $\tau_{0}$, the three-stage reference function $s(t)$ is

$$
\begin{aligned}
& s_{I}(t)=\frac{1}{\tau-\tau_{0}}\left(-\frac{5 t^{8}}{2 \tau_{0}^{7}}+\frac{10 t^{7}}{\tau_{0}^{6}}-\frac{14 t^{6}}{\tau_{0}^{5}}+\frac{7 t^{5}}{\tau_{0}^{4}}\right) \\
& s_{I I}(t)=\frac{1}{\tau-\tau_{0}}\left(t-\frac{\tau_{0}}{2}\right) \\
& s_{I I I}(t)=1+\frac{1}{\tau-\tau_{0}}\left(\frac{5(\tau-t)^{8}}{2 \tau_{0}^{7}}-\frac{10(\tau-t)^{7}}{\tau_{0}^{6}}+\frac{14(\tau-t)^{6}}{\tau_{0}^{5}}-\frac{7(\tau-t)^{5}}{\tau_{0}^{4}}\right)
\end{aligned}
$$

Given $\tau=40 \mathrm{~s}$ and $\tau_{0}=10 \mathrm{~s}$ for the present maneuver, the reference function $s(t)$ of (29), and its first and second time derivatives are as in Fig. 4.

Selected results of the inverse dynamics simulation, obtained as the solution to the governing DAEs represented in (21) by using the integration scheme of (24) for $\Delta t=0.1 \mathrm{~s}$, are seen in Fig. 5. The execution of the load specified motion requires adequate changes in the bridge rotation, the trolley position on the bridge and the rope length, respectively, 
Fig. 4 The reference function and its time derivatives
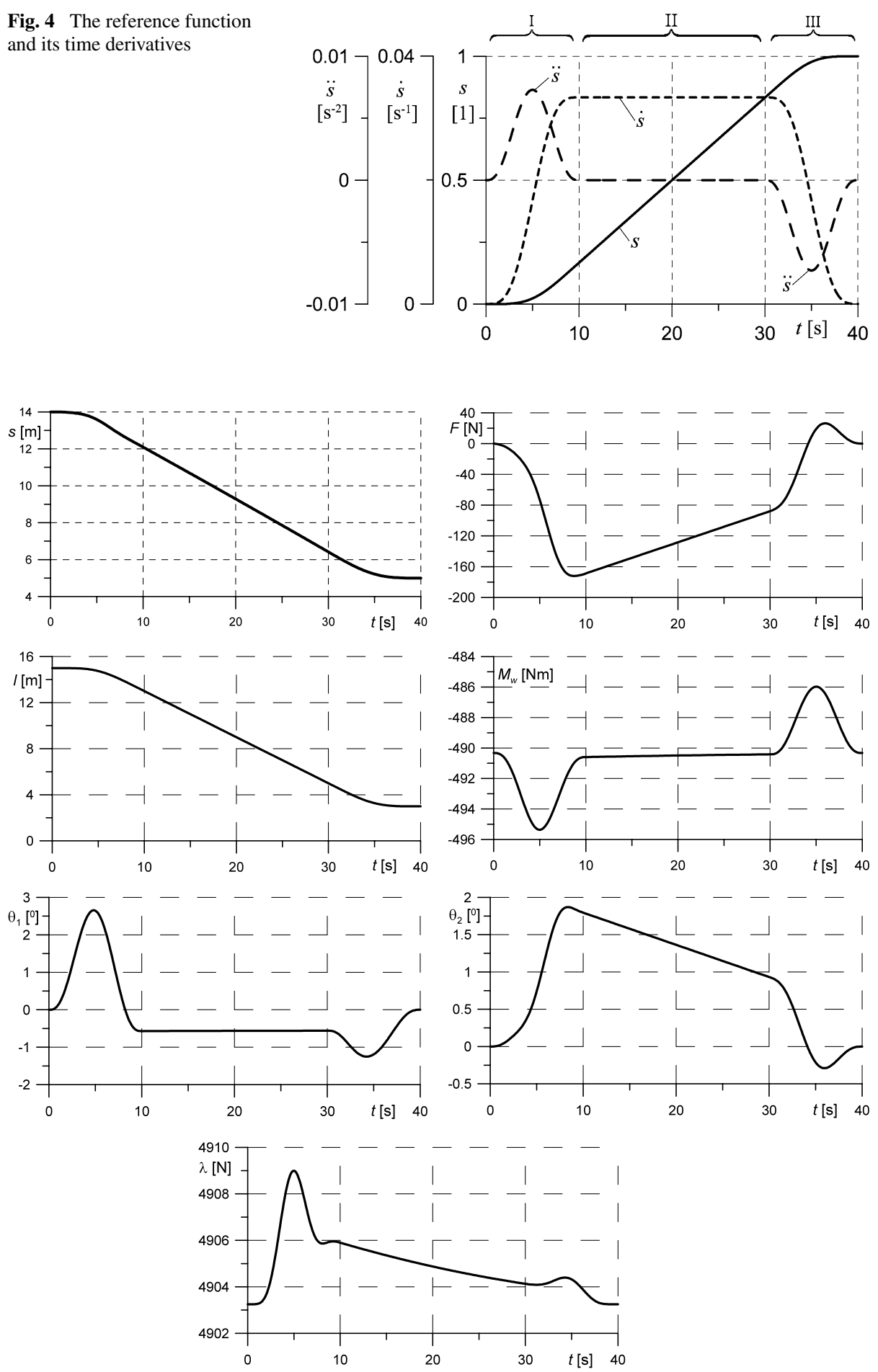

Fig. 5 Motion and control of the robot executing the load specified motion 
$\varphi_{\mathrm{d}}(t), s_{\mathrm{d}}(t)$, and $l_{\mathrm{d}}(t)$, enforced by the appropriate control reactions $M_{\mathrm{bd}}(t), F_{\mathrm{d}}(t)$, and $M_{\mathrm{wd}}(t)$. The variations of the tensile force in the rope $\lambda_{\mathrm{d}}(t)$ are also obtained. The swing angles $\theta_{1}$ and $\theta_{2}$, defined in Fig. 1a, were then calculated from the solution $\mathbf{p}_{\mathrm{d}}(t)$ and the load motion specification $\mathbf{r}_{\mathrm{d}}(t)$.

\section{Conclusion}

The advantages in modeling and simulation of cranes using the dependent coordinates over the independent variable formulation are the following: $(\bullet)$ both the dynamic equations of motion and the servo-constraint equations are considerably simpler, resulting in much simpler governing equations of the inverse simulation problem, $(\bullet)$ the actual number of the governing equations is smaller, $3 m+1$ compared to $5 m-2$, and $(\bullet)$ more physical insight into the problems solved is brought by directly involving the tensile force in the rope as one of the variables of the analysis, which is not the case of the independent variable formulation. Evidently, the numerical simulations based on the dependent and independent variable formulations lead to exactly the same results.

Open Access This article is distributed under the terms of the Creative Commons Attribution Noncommercial License which permits any noncommercial use, distribution, and reproduction in any medium, provided the original author(s) and source are credited.

\section{References}

1. Spong, M.W.: Underactuated mechanical systems. In: Siciliano, B., Valavanis, K.P. (eds.) Control Problems in Robotics and Automation. Lecture Notes in Control and Information Sciences, vol. 230, pp. 135150. Springer, London (1998)

2. Fantoni, I., Lozano, R.: Non-linear Control for Underactuated Mechanical Systems. Springer, London (2002)

3. Sahinkaya, M.N.: Inverse dynamic analysis of multiphysics systems. Proc. IME, J. Syst. Control Eng. 218, 13-26 (2004)

4. Fliess, M., Lévine, J., Martin, P., Rouchon, P.: Flatness and defect of nonlinear systems: introductory theory and examples. Int. J. Control 61, 1327-1361 (1995)

5. Blajer, W., Kołodziejczyk, K.: A geometric approach to solving problems of control constraints: theory and a DAE framework. Multibody Syst. Dyn. 11, 343-364 (2004)

6. Rouchon, P.: Flatness based control of oscillators. Z. Angew. Math. Mech. 85, 411-421 (2005)

7. Abdel-Rahman, E.M., Nayfeh, A.H., Masoud, Z.N.: Dynamics and control of cranes: a review. J. Vib. Control 9, 863-908 (2003)

8. Omar, H.M.: Control of gantry and tower cranes. PhD Thesis, Virginia Polytechnic Institute and State University, Blacksburg, Virginia, USA (2003)

9. Lee, H.H.: Motion planning for three-dimensional overhead cranes with high-speed load hoisting. Int. J. Control 78, 875-886 (2005)

10. Blajer, W., Kołodziejczyk, K.: Dynamics and control of rotary cranes executing a load prescribed motion. J. Theor. Appl. Mech. 44, 929-948 (2006)

11. Blajer, W., Kołodziejczyk, K.: A DAE formulation for the dynamic analysis and control design of cranes executing prescribed motions of payloads. In: García Orden, J.C., Goicolea, J.M., Cuadrado, J. (eds.) Multibody Dynamics. Computational Methods and Applications. Computational Methods in Applied Sciences, vol. 4, pp. 91-112. Springer, Dordrecht (2007)

12. Blajer, W., Kołodziejczyk, K.: Control of underactuated mechanical systems with servo-constraints. Nonlinear Dyn. 50, 781-791 (2007)

13. Kirgetov, V.I.: The motion of controlled mechanical systems with prescribed constraints (servoconstraints). J. Appl. Math. Mech. 21, 433-466 (1967)

14. Bajodah, A.H., Hodges, D.H., Chen, Y.-H.: Inverse dynamics of servo-constraints based on the generalized inverse. Nonlinear Dyn. 39, 179-196 (2005) 
15. Rosen, A.: Applying the Lagrange method to solve problems of control constraints. ASME Trans. J. Appl. Mech. 66, 1013-1015 (1999)

16. Woernle, Ch.: Control of robotic systems by exact linearization methods. In: Maisser, P., Tenberge, P. (eds.) Proc. of the First International Symposium on Mechatronics (ISoM 2002), pp. 207-218. Advanced Driving Systems, Chemnitz (2002)

17. Heyden, T., Woernle, Ch.: Dynamics and flatness-based control of a kinematically underdetermined cable suspension manipulator. Multibody Syst. Dyn. 16, 155-177 (2006)

18. Aschemann, H.: Optimale Trajektrienplanung sowie modelgestützte Steuerung für einen Brückenkran. Fortschritt-Berichte VDI, Reihe 8: Meß-, Steuerungs- und Regelungstechnik, Nr. 929. Düsseldorf, Germany (2002)

19. Blajer, W., Kołodziejczyk, K.: Motion planning and control of gantry cranes in cluttered work environment. IET Control Theory Appl. 1, 1370-1379 (2007)

20. Ascher, U.M., Petzold, L.R.: Computer Methods for Ordinary Differential Equations and DifferentialAlgebraic Equations. SIAM, Philadelphia (1998)

21. Campbell, S.L., Gear, C.W.: The index of general nonlinear DAEs. Numer. Math. 72, 173-196 (1995)

22. Gear, C.W.: An introduction to numerical methods for ODEs and DAEs. In: Haug, E.J., Deyo, R.C. (eds.) Real-Time Integration Methods for Mechanical System Simulations. NATO ASI Series, vol. F69, pp. 115-126. Springer, Berlin (1990)

23. Petzold, L.R.: Methods and software for differential-algebraic systems. In: Haug, E.J., Deyo, R.C. (eds.) Real-Time Integration Methods for Mechanical System Simulations. NATO ASI Series, vol. F69, pp. 127-140. Springer, Berlin (1990)

24. Blajer, W.: A geometric unification of constrained system dynamics. Multibody Syst. Dyn. 1, 3-21 (1997) 